\title{
INSIGHT INTO THEORY-EXPERIMENT COMPARISONS OF WAKE MEASUREMENTS IN THE PLASMASPHERE
}

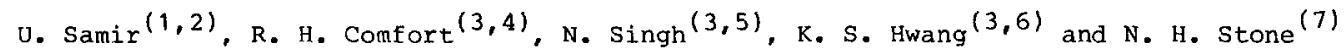

1. Space Physics Research Laboratory, University of Michigan, Ann Arbor, MI 48109

2. Department of Geophysics and Planetary Sciences, Tel-Aviv University, Israel

3. Center for Space Plasma and Aeronomic Research, University of Alabama in Huntsville, Huntsville, AL 35899

4. and Departmenl of Pliysics

5. and Department of Electrical \& Computer Engineering

6. and Department of Mechanical Engineering

7. Space Science Iaboratory, NASA/Marshal1. Space Flight Center, Huntsville, AL 35812

$$
\text { (Camera-realv cony received } 17 \text { thy 1989) }
$$

\begin{abstract}
Calculated results from a modified one-dimensional time-dependent plasma expansion model are compared with wake measurements of low energy $\mathrm{H}^{+}$ions made in the plasmasphere which consists of $77-90 \% \mathrm{H}^{+}$and $23-10 \% \mathrm{H}_{e}^{+}$. The variation of the theoretical-to-experimental results with the $\mathrm{H}^{+}$ionic Mach number $\left(\mathrm{S}_{\mathrm{H}+}\right)$ in the range $0.5<\mathrm{S}_{\mathrm{H}+}<1.2$ is shown. It is found that the theoretical model used overestimates the particle flux in the wake by factors of 2 to 6 . Possible causes for these differences are examined. Discussions regarding: (1) the shortcomings of using one-dimensional models and other simplifications commonly used in theoretical modeling, (2) the use of measurements from probes having finite angular acceptance in wake studies, and (3) a relative comparison between a three-dimensional neutral approximation and the modified one-dimensional model used here are given. This comparison shows that for the plasmaspheric parameters used here, the application of a neutral particle model yields reasonable results, since the ion thermal motion rather than the local electric field dominates the filling of the wake (particularly for weakly charged bodies). These discussions provide additional insight into such theory-experiment comparisons and should be of practical use.
\end{abstract}

\section{INTRODUCTION}

Gurevich et al. (1966) suggested that plasma expansion processes may have a direct relation to the problem of the flow of a rarefied plasma past a rapidly moving object. Further plasma expansion studies were reported in Gurevich et al. (1968, 1973), Gurevich and Meshcherkin (1981a,b), Crow et al. (1975), Lonngren (1977), Lonngren and Hershkowitz (1979), Denavit (1979), Sack and Schamel (1985) to cite but a few. More recently (Samir et al., 1983) it has been suggested that plasma expansion processes, such as the propagation of a rarefaction wave, the motion of an ion-front, and ion acceleration, may take place in the wake region behind a body (artificial and/or natural) moving in a space plasma. Some evidence in this regard has been provided by laboratory space simulation experiments (Eselevich and Fainshtein, 1981; Chan et al., 1984; Chan, 1986; Wright et al., 1985, Wright et al., 1986; Raychaudheri et al., 1986). Attempts to apply plasma expansion to the interpretation of several space plasma processes in the terrestrial ionosphere and magnetosphere have also been made (Singh and Schunk, 1982, 1983, 1985; Diebold et al., 1987a,b; Kozima et al., 1988; Wright, 1988). Based on these (Gurevich and Meshcherkin, 1981a; Singh and schunk, 1983) and on some laboratory results (Wright et al., 1985, 1986; Raychaudheri, 1986), it was suggested that plasma expansion models could explain, at least semi-quantitatively, the spatial distribution of ions and electrons in the wake of an artificial satellite orbiting the earth.

It is the objective of this note to test the above suggestion and provide results of a theory-experiment comparison, which employed a modified one-dimensional time-dependent counterstreaming plasma expansion model (PEM) based on Singh and Schunk, 1983; Singh et al., 1986 and on in-situ measurements of the distribution of ions in the very near wake of the Dynamics Explorer 1 (DE-1) satellite. By means of previous comparisons of the data with neutral approximation models (Samir et al., 1986), we can also assess the usefulness of the PEM relative to the neutral models for this flow regime. The measurements used are from the 
Retarding Ion Mass Spectrometer--RIMS radial head, which samples the incoming particle flux in the spin plane of the DE-1 satellite with a limited aperture detector, having an effective half angle acceptance cone of $30^{\circ}$ (Comfort et al., 1985). The peM used has already demonstrated its applicability in other areas of space plasma physics and was modified to partially account for the current collection in the wake situation. Originally, the PEM (singh et al., 1936) used counterstreaming two-ion-species plasma, but in the present comparison we use the results for only the major ionic constituent, $\mathrm{H}^{+}$. Details of the numerical simulation technique and the method of analysis of the RIMS in-situ measurements are given elsewhere (Singh and Schunk, 1983; Comfort et al., 1982, 1985).

\section{THE EXPERIMENTAL. DATA AND THE THEORETICAL MODEL}

Table 1 gives some relevant body and plasma parameters, as well as measured and calculated ion depletion ratios. Columns \#1 and \#2 give the $\mathrm{H}^{+}$ambient temperature and density, respectively. Column \#3 gives the normalized ( $\left.\phi_{\mathrm{N}}=\mathrm{e} \phi_{\mathrm{S}} / \mathrm{kT}\right)$ potential of the satelite ( $\phi_{s}$ is the value of satellite potential with respect to the local plasma potential as deduced from the specific ion $\left(\mathrm{H}^{+}\right)$analysis) (Samir et al., 1986). The fourth column gives the parameter $\mathrm{R}_{\mathrm{Di}}=\mathrm{R}_{\mathrm{O}} / \lambda_{\mathrm{Di}}$, the normalized body size $\left(\mathrm{R}_{\mathrm{O}}=\right.$ the effective radius of the satellite and $\lambda_{\mathrm{Di}}=$ the ionic ambient Debye length). Column $\# 5$ gives the ionic ( $H^{+}$) velocity number, $S$ $=\left(\mathrm{MV}_{\mathrm{S}}^{2} / 2 \mathrm{kT}\left(\mathrm{H}^{+}\right)\right)^{1 / 2}$. The last two columns present, respectively, the observed and theoretical ionic depletion ratios for $\mathrm{H}^{+}$, as described below.

TABLE 1. Some relevant body and plasma parameters and ion depletion ratios for $\mathrm{H}^{+}$

\begin{tabular}{|c|c|c|c|c|c|c|c|}
\hline Case \# & $\begin{array}{l}\frac{(1)}{T\left(H^{+}\right)} \\
\left({ }^{\circ} \mathrm{K}\right)\end{array}$ & 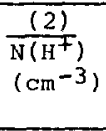 & $\frac{(3)}{\phi_{N}\left(H^{+}\right)}$ & $\frac{(4)}{\mathrm{R}_{\mathrm{Di}}}$ & $\frac{(5)}{S\left(H^{\mp}\right)}$ & $\begin{array}{c}\frac{(6)}{\operatorname{Exp}} \\
\alpha^{\alpha}-2 \\
\left(10^{-2}\right)\end{array}$ & $\begin{array}{c}\frac{(7)}{\text { Theory }} \\
\left(10^{\alpha}\right)\end{array}$ \\
\hline $1(6) *$ & 2362 & 3273 & -1.44 & 10.46 & 1.18 & 1.61 & 3.13 \\
\hline $2(5)$ & 2323 & 3398 & -2.10 & 10.73 & 1.14 & 2.18 & 4.10 \\
\hline $3(10)$ & 3923 & 3680 & -1.46 & 9.05 & 0.97 & 1.43 & 6.32 \\
\hline $4(9)$ & 4073 & 3621 & -0.98 & 8.89 & 0.97 & 1.25 & 6.05 \\
\hline $5(13)$ & 4055 & 3702 & -0.85 & 8.74 & 0.88 & 1.92 & 8.60 \\
\hline $6(15)$ & 4360 & 2898 & -0.01 & 7.66 & 0.78 & 2.64 & 13.4 \\
\hline $7(17)$ & 4508 & 1498 & 0.08 & 5.27 & 0.68 & 3.09 & 14.2 \\
\hline $8(18)$ & 5232 & 1065 & 0.56 & 4.15 & 0.60 & 3.07 & 18.0 \\
\hline $9(24)$ & 5431 & 2124 & 0.07 & 5.46 & 0.54 & 7.67 & 22.5 \\
\hline $10(25)$ & 5806 & 1386 & 0.20 & 4.30 & 0.49 & 7.33 & 24.6 \\
\hline
\end{tabular}

*Numbers in () are case numbers from Samir et al. (1986).

As seen from the table, the plasma is highly rarefied and the temperatures are in the $1 \mathrm{eV}$ range. The body we are treating is considered, small $\left(\mathrm{R}_{\mathrm{Di}}<10\right)$, as is its potential $\left(\left|\phi_{N}\right|<2\right)$. Essentially we are dealing with an $\mathrm{H}^{+}$plasma, with a minor constituent (He ${ }^{+}$) being $10 \%-23 \%$ of the total ionic concentration. Hence, the experimental values and the computations reported here refer to the II $^{+}$component only. As discussed by Gurevich et al. (1973) theoretically, by Samir et al. (1986) based on in-situ measurements, and recently by Wright (1988) based on laboratory simulation experiments, when the lighter ion species is the major ion, each ionic species behaves approximately independently. Therefore, presenting only the $\mathrm{H}^{+}$ion adequately depicts the situation in the very near wake.

The ion depletion in the wake, $\alpha$, is defined as the ratio of the ion current in the wake to the ion current in the satellite's ram direction. More specifically, column \#6 gives the experimental values for the ratio $I_{H+}$ (wake) $/ I_{H+}(r a m)=I_{H^{+}}\left(180^{\circ} \pm 15^{\circ}\right) / I_{H+}\left(0^{\circ} \pm 15^{\circ}\right)$ as measured by the RIMS probe. Column $\# 7$ yields the corresponding flux ratios computed from the plasma expansion model (PEM) of Singh et al. (1986) and the modification discussed below. It should be noted that the experimental and theoretical ratios given in columns \#6 and \#7 can serve as quantitative measures of the degree of disturbance caused by the satellite's interaction with its environmental space plasma. A diagram of the physical problem addressed by the calculation is shown in Fig. 1a, in which we consider an initial plasma void of width $2 R_{s}$, where $R_{S}$ is the spacecraft dimension, which is approximated to be a cylinder of radius $R_{S}$. In this model, we assume that the initial plasma void is planar; it occupies the spatial region $-R_{s}<x<R_{s}$; and it extends to $\pm^{\infty}$ in the $y$-direction. In effect the model replaces the spherical satelitite by a plate of width $2 R_{S}$. The ambient plasma has a velocity $V_{S}$ in the 

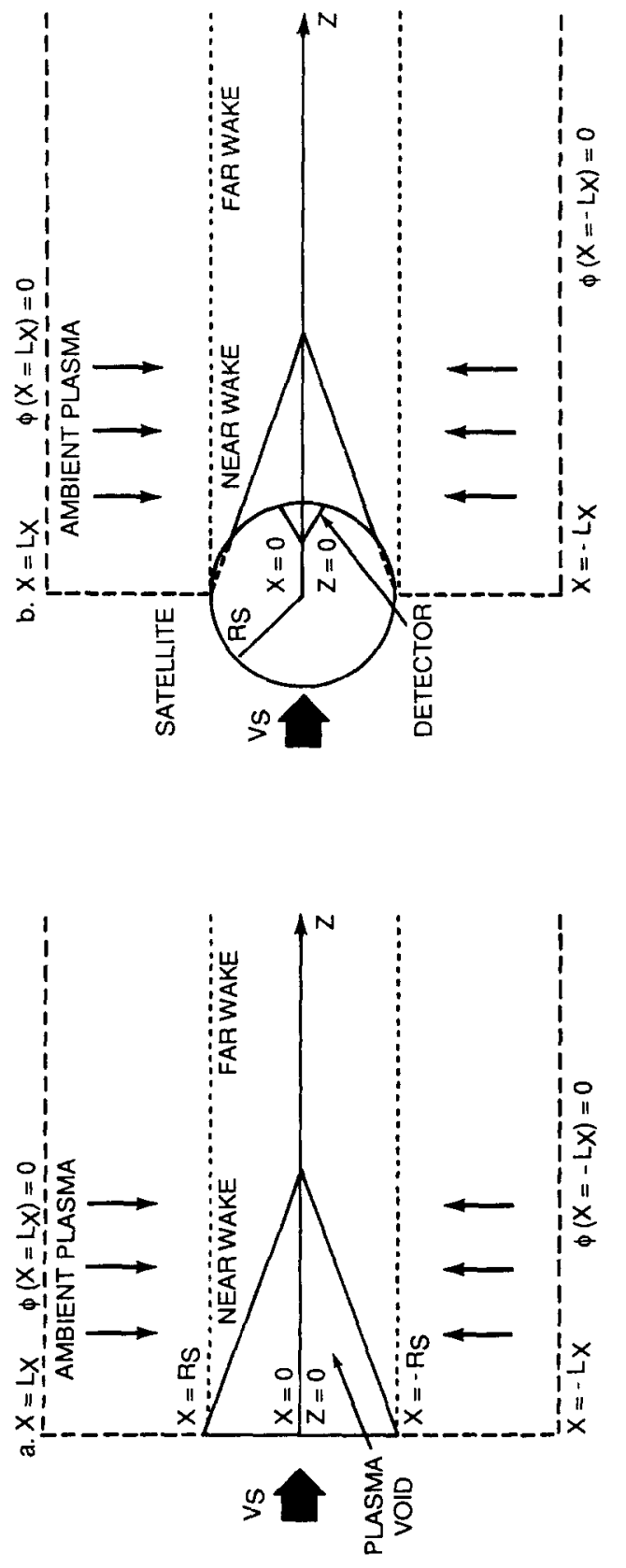

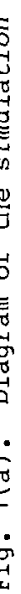


rest frame of the satellite; and it expands into the void from both sides of the wake, as indicated by upward and downward arrows in Fig. 1 a.

The time-dependent plasma expansion into the wake is followed by solving the vlasov equation for the major $\mathrm{H}^{+}$and minor $\mathrm{He}^{+}$ions in the ambient plasma along with the Poisson equation. Electrons are assumed to obey the Boltzmann relation. As the plasma expands along the $x$-direction into the plasma void, it also flows along the $z$ axis with the velocity $v_{s}$. Therefore, the time variable in the expansion is related to the axial distance $z$ according to $z=v_{s} t$. Thus, when the plasma reaches the detector by expanding in the $x$-direction and flowing with velocity $V_{S}$ in the z-direction, $z=R_{S}$ and $t=R_{s} / V_{s}$.

Starting with $t=0$, the expansion is followed until the time given above. This yields the ion velocity distribution function $\mathrm{g}\left(\mathrm{V}_{\mathrm{x}}\right)$. Note that while the expansion in this onedimensional calculation is in the $x$-direction (Fig. 1a), the detector axis (in the observations) is in the $z$-direction with a finite angular acceptance (Fig. 1b). In order to estimate the portion of those particles reaching the $\mathrm{X}=0$ axis which enter the detector, the following procedure is used. The velocity distribution $f\left(V_{z}\right)$ of the ions remains a flowing Maxwellian, as in the ambient plasma. Taking the first moment of the composite distribution function

$$
F\left(V_{x}, v_{z}\right)=N_{0} N 1 /\left[\overline { l } _ { t } \operatorname { O e x p } \left(-\left(v_{z}-v_{s}\right)^{2} / v_{t}^{2} \lg \left(v_{x}\right)\right.\right.
$$

over the velocity range $-B<V_{z}<0$, an estimate for the ion flux, $q_{\text {wake }}$ in the wake at the detector axis can be obtained. Here $v_{t}$ is the ion thermal speed. Column \#7 gives the ratio $q_{\text {wake }} / q_{r a m}$, where the ram flux $q_{\text {ram }}$ is obtained by integrating $F\left(v_{x}\right.$, $\left.v_{z}\right)$ over the range $0<V_{z}<B$. We note that this procedure is tantamount to using a neutral approximation for the $v_{z}^{z}$ component, and it simulates the response of an "open" aperture detector with $90^{\circ}$ halfangle acceptance (cf. Samir et al., 1987). Note that "neutral approximation" indicates that ions are treated as if they were neutral particles in the trajectory calculations.

The degree of agreement/difference between theory and experiment is shown in Figure 2, where the ratio a (theory)/a(experiment) (values from Table 1) is plotted versus ( $S_{H}+$ ). The figure shows that the theory overestimates the flux (or current) collected in the maximum wake reqion by factors of 2 to 6 . Although it appears that the range of $\mathrm{S}\left(\mathrm{H}^{+}\right)$used here is fairly narrow $\left(0.5<\mathrm{S}\left(\mathrm{H}^{+}\right)<1.2\right)$, this is the range characteristic of the terrestrial plasmasphere. The same applies to $R_{D i}$ and $d_{N}$.

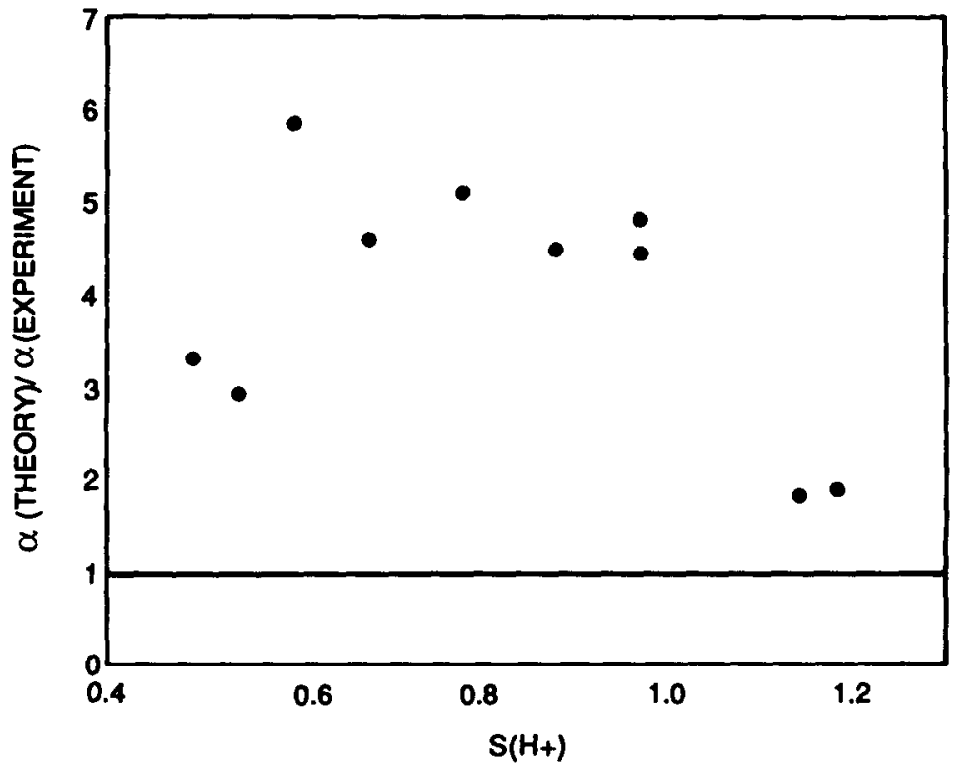

Fig. 2. Ratio of theoretical to observed ion depletion ratios vs. ionic Mach number. 


\section{COMPARISON OF IN-SITU DATA MITH THEORETICAL MODELS}

In this comparison between theory and experiment, there are several sources for the differences seen in Figure 2. These may be grouped into three categories: (i) the model used is essentially one-dimensional and time-dependent; (ii) body geometry is simplified; and (iii) body potential is not included in the calculation. Note, however, that space-charge potential is considered for the expansion in the $x$-direction, and no self-similar approximations are used.

Intuitively, it would seem that a three-dimensional calculation, which includes fluxes in the $y$ - and $z$-directions, in addition to those in the $x$-direction, would result in higher ion fluxes in the spacecraft wake. However, with the compensation (or: modification) introduced above for the $z$-direction, it is not clear that in a self-consistent treatment of the wake space-charge potential, as is used in the PEM, a higher flux would necessarily result for a proper three-dimensional treatment, due only to the added dimensionality of the calculation. Some of the considerations discussed below, indicate other associated problem areas which need to be addressed when comparing measurements with theoretical models, and which may by themselves account for (at least) a good part of the difference.

In the one-dimensional treatment used here the fluxes calculated from the plasma expansion process correspond to the x-direction (transverse to the wake), whereas the observations were made with the detector pointed in the z-direction (parallel to the wake). As described above, this was compensated by computing the fraction of the flux moving toward the body under a neutral particle approximation. The use of the latter approximation is not unreasonable for a plasmaspheric flow regime.

The procedure adopted to make this compensation has two opposing consequences. On the one hand, this $z$-component $f l u x$ is probably underestimating the collected current since it ignores the space charge potential in the satellite wake. The space-charge potential is taken into account in the calculation of the $x$-component of the flux.

Opposing this is a consequence of the "effective aperture" angle which is implicit in the mothod used to compute the ionic depletion in the wake for the PEM results. All particles with $v_{z}$ components toward the spacecraft are counted in both ram and wake directions for the PEM results, so that effectively the resulting ionic depletion ratio corresponds to that from a detector with an "open" aperture, i.e., half-angle acceptance of $90^{\circ}$. The RIMS detector which made the observations, has an effective half-angle acceptance of $30^{\circ}$ (Comfort et al., 1982, 1985; Samir et al., 1986, 1987). The quantitative importance of this difference on ionic depletion ratios have been demonstrated and discussed in detail elsewhere (Samir et al., 1987). The result is that ionic depletion ratios from measurements by a $30^{\circ}$ detector are smallex than those from a $90^{\circ}$ detector. In this comparison then, this effect causes an overestimate of the ion depletion ratio by the PEM.

Another factor which has the effect of producing a larger wake flux than would be observed is the body boundary used in the calculation vis-a-vis the actual body boundary (see Figure 1b). In the calculation, the body creating the wake is a flat plate at $z=0$, whereas the actual body corresponds more closely to a cylinder. Since the region immediately behind the body will have the largest negative space charge potential (Gurevich and Meshcherkin, 1981a; Singh and Schunk, 1983), the calculation of fluxes (Fig. 1a) is based on a large negative potential occupying the spatial region which, in the correct geometry, should be occupied by the body (Fig. 1b). This region thus draws in more ions in the calculation than it does in reality, resulting in an overestimate of the ion depletion ratio. A second effect of body geometry is that some particle trajectories which in the calculation contribute to the population on the $x=0$ axis near $z=R_{s}$ would be intercepted by the spacecraft and never arrive there. This also contributes to the theoretical overestimate of the ion depletion ratio.

with regard to the neglect of the body potential in the PEM calculations, from Table 1 we see three trends occurring simultaneously in the observational conditions. As ionic Mach number (S) increases, the normalized body potential $\phi_{\mathrm{N}}$ is becoming more negative (although still small), and the body size $R_{D i}$ is increasing. These relate to changing plasma properties and spacecraft velocity along the spacecraft orbit (Samir et al., 1986). The theoretical calculations take into account variations of $S$ and $R_{D_{i}}$, but not those of $\phi_{N}$. When $\phi_{N}<0$, more ions are expected to be collected by a probe on the spacecraft, and fewer particles when $\phi_{N}>0$. Hence, we expect the largest underestimate of particle fluxes in the theoretical calculations to occur with the most negative values of $\phi_{N}{ }^{*}$ This is consistent with the trend seen in Figure 2. We note that the average difference between theory and observation in Figure 2, is by a factor of about 5, whereas in Samir et al. (1986) it was shown that a three-dimensional neutral approximation model with a spherical geometry differs from the experimental measurements by an average factor of about 2.5 .

Hence, for the determination of the ion collection in the near-wake in the flow regime of interest here, a one-dimensional self-consistent plasma expansion model even modified as 
explained above has no advantage over a neutral approximation model. It appears to us that for plasmaspheric conditions, omitting the overall field potential in the model may be less significant than omitting other factors.

It is possible that for a plasmaspheric flow regime a three-dimensional plasma expansion model, such as used in Murphy and Katz (1987), which uses simplifying assumptions to predict wake densitles, may be applicable. In the latter model, known also as the POLAR code (Katz et al., 1985) a self-similar treatment is used for the particles in the region intermediate between the "undisturbed region" and the region of "higher rarefaction" (see Figure 3), and a "neutral approximation" was used for particles in the region of higher rarefaction. The above plasma expansion model was used for ionospheric conditions (shuttle-spacelab 2 orbit), but at a distance of several radii downstream of the body, and a theory-experiment agreement of less than $20 \%$ is claimed. For the region immediately behind the body, which is the case of the present note, the treatment used in Fig. 3 should not be expected to yield results much different from the simple neutral approximation treatment used in Samir et al. (1986). Further, it is noted that for small body potentials and a flow regime such as the plasmasphere, a neutral approximation is not unreasonable. Regarding the yular code, it should be noted that in the plasmaspheric flow regime, this code goes to the neutral limit and is thus valid as a neutral approximation. It is possible that for a flow regime in space with higher Mach numbers, where a simple neutral approximation is not applicable, the POLAR code may be useful.
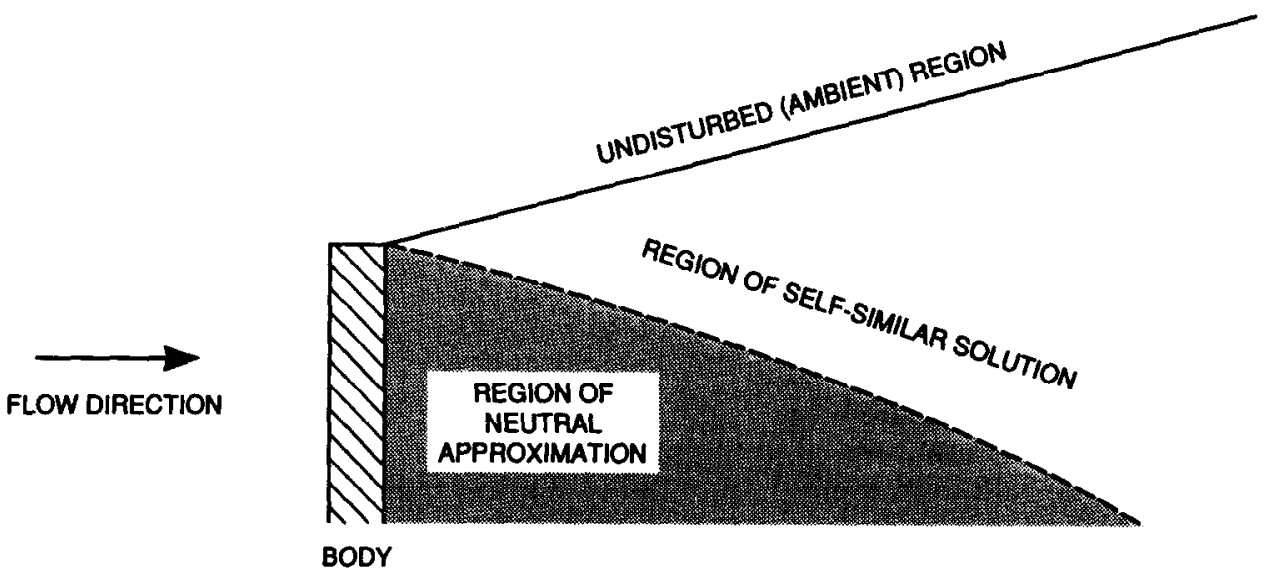

Fig. 3. The POLAR code treatment of the Shuttle (Spacelab-2) wake. After Murphy and Katz (1987).

At the present time it appears that a self-consistent one-dimensional (x-axis) plasma expansion model, modified to account for current collection in the z-direction via a neutral approximation treatment, yields results (for theory-expeximent comparisons) which are no better than a simplified three-dimensional neutral approximation treatment (Samir et al., 1986). Considering the effective low magnitudes of body potential in the plasmaspheric flow regime (Table 1), it appears that in order to achieve a better agreement between theory and experiment, attention should be given to the body geometry and the dimensionality in the models. Of course, using a three-dimensional self-consistent model which accounts for body size, geometry, and potential under realistic conditions should be sought. That, however, has not yet been achieved, despite fairly extensive efforts throughout the last three decades

The competition between body and plasma parameters in determining the wake structure is of great importance to this area of investigation. This is particularly so for the very near wake region. Body geometry should be less significant for the far-wake region. For an ionospheric flow regime, where the body potentials are significantly more negative, the $\phi_{N}$ parameter may be in competition with $s$, and the body geometry may be of less importance, particularly at larger distances down the wake. 


\section{CONCLUSION}

At the present time, based on the plasmaspheric measurements used here, it is clear that a simple plasma expansion model, e.g., one-dimensional (although self-consistent), used here does not quantitativeiy represent the distribution of low energy ions in the maximum rarefaction region of the very near wake. Causes for the difference between theory and measurements may not be only or even mainly due to the addition of two more dimensions in the plasma expansion modeling, and to achieve the latter is quite complicated and costly. The three-dimensional model that now exists (Katz et al., 1985; Murphy \& Katz, 1987) is not significantly (and conceptually) superior to a simple three-dimensional neutral approximation model for these plasmaspheric conditions. For ionospheric plasmas, the relative significance of other parameters (e.g., the normalized potential) may be greater.

\section{ACKNOWLEDGEMENTS}

Ur. U.S. acknowledges support under NASA grant NGK-23-005-320, and the interest of Drs. S. Shawhan and T. Eastman of NASA Headquarters. Drs. R.H.C. and N.S. were partially supported by NASA/MSFC grant NAG8-058 and Drs. K.S.H. and N.S. were supported in part by NASA/MSFC contract NAS8-37107. The authors acknowledge the comments made by Dr. K.H. Wright.

\section{REFERENCES}

Chan, C. (1986) Laboratory experiments on plasma expansion, in Ion Acceleration in the Magnetosphere and Ionosphere. Geophys. Mono. 38, 249.

Chan, C., N. Hershkowitz, A. Ferreira, T. Intrator, B. Nelson, and K. Lonnegren (1984) Experimental observations of self-similar plasma expansion. Phys. Fluids $27,266$.

Comfort, R. H., C. R. Baugher, and C. R. Chappell (1982) Use of the thin sheath approximation for obtaining ion temperatures from the DE-1 limited aperature RPA. J. Geophys. Res. 87, 5109.

Comfort, R. H., J. H. Waite, Jr., and C. R. Chappell (1985) Thermal ion temperatures from the retarding ion mass spectrometer on DE-1. J. Geophys. Res. 90, 3475, 1985.

Crow, J. E., P. L. Auer, and J. E. Allen (1975) The expansion of a plasma into a vacuum. J. Plasma Phys. 14, 65 .

Denavit, J. (1979) Collisionless plasma expansion into a vacuum. Phys. Fluids 22, 1384.

Diebold, D., N. Hershkowitz, T. Intrator, and A. Bailey (1987a) Self-similar potential in the near wake. Phys. Fluids 30, 579.

Diebold, D., $\vec{N}$. Hershkowitz, and $S$. Eliezer (1987b) Rarefaction shock in the near wake. Phys. Fluids 30, 3308.

Eselevich, V. G., and V. G. Fainshtein (1981) Expansion of a collisionless plasma into vacuum. Sov. J. Plasma Phys. 7, 271.

Gurevich, A. V., and A. P. Meshcherkin (1981a) Ion acceleration in an expanding plasma, Sov. Phys. IETP 53, 937.

Gurevich, A. V., and A. P. Meshcherkin (1981b) Jump discontinuity on the front of a rarefaction wave front in a plasma. Sov. Phys. JETP 54, 688.

Gurevich, A. V., L. V. Paryiskaya, and L. P. Pitaevsky (1966) Self-similar motion of rarefied plasma. Sov. Phys. JETP 22, 449.

Gurevich, A. V., L. V. Paryiskaya, and L. P. Pitaevsky (1968) Self-similar motion of a low density plasma II. Sov. Phys. JETP 27, 476.

Gurevich, A. V., L. V. Paryiskaya, and L. P. Pitaevsky (1973) Ion acceleration upon expansion of a rarefield plasma. Sov. Phys. JETP 36, 274.

Katz, I, E. Parks, and K. H. Wright (1985) "A model of the plasma wake generated by a large object." IFFE Trans. Nuc. Sci., NS-32, 4092.

Kozima, H., H. Shimizu, K. Yamada, T. Mieno, and K. Yamagiwa (1988) Self-similar flow and related phenomena of plasmas around obstacles. J. Phys. Soc. Japan 57, 1136.

Lonngren, K. E. and N. Hershkowitz (1979) A note on plasmas expansion into a vacuum. IEEE Trans. Plasma Sci. PS-7, 107.

Lonngren, K. E. (1977), Self-similar solution of plasma equations. Proc. Indian Acad. Sci. 86A, 125 .

Murphy, G. B., and I. Katz (1987) "The polar code wake model: comparison with in-situ observations," submitted to J. Geophys. Res..

Raychaudhuri, S., J. Hill, H. Y. Chang, E. K. Tsikis, and K. E. Lonngren (1986) An experiment on the plasma expansion into a wake. Phys. Fluids 29, 289.

Sack, Ch., and $H$. Schamel (1985) Evolution of a plasma expanding into vacuum. Plasma Phys. Contr. Fus. 27, 717. 
Samir, U., K. H. Wright, Jr., and N. H. Stone (1983) The expansion of a plasma into a vacuum: basic phenomenon and processes and applications to space plasma physics Rev. Geophys. 21, 1631 .

Samir, U., R. H. Comfort, C. R. Chappell, and N. H. Stone (1986) Observations of low-enery ion in the wake of a magnetospheric satellite. J. Geophys. Res. 91, 5725.

Samir, U., R. H. Comfort, N. H. Stone, and K. H. Wright, Jr. (1987) Intercomparison among plasma wake models for plasmaspheric and ionospheric conditions. Planet. Space Sci. 35, 1477.

Singh, N., and R. W. Schunk (1982) Numerical calculations relevent to the initial expansion of the polar wind. J. Geophys. Res. 87, 9154.

Singh, N., and R. W. Schunk (1983) Numerical simulations of counterstreaming plasmas and their relevance to interhemispheric flows. J. Geophys. Res. 88, 7867.

Singh, N., and R. W. Schunk (1985) Temporal behavior of density perturbation in the polar wind. J. Geophys. Res. 90, 6487.

Singh, N., H. Thiemann, and R. W. Schunk (1986) Studies on counterstreaming plasma expansion. Physica Scripta 33, 355.

Wright, K. H., Jr., A study of single and binary ion plasma expansion into laboratory generated plasma wakes, NASA Contractor Report 4125, February, 1988.

wright, K. H., Jr.. N. H. Stone, and U. Samir (1985) Study of plasma expansion phenomena in laboratory generated plasma wakes: preliminary results. J. Plasma Phys. 33, 71.

wright, K. H., Jr., D. E. Parks, I. Katz, N. H. Stone, and U. Samir (1986) More on the expansion of a collisionless plasma into the wake of a body. J. Plasma Phys. 35, 119. 